Post-print version (peer reviewed, author accepted manuscript), please cite the published article as follows:

Favrod, O., da Cruz, J.R., Roinishvili, M., Berdzenishvili, E., Brand, A., Figueiredo, P., Herzog, M.H. and Chkonia, E., 2019. Electrophysiological correlates of visual backward masking in patients with major depressive disorder. Psychiatry Research: Neuroimaging, 294, p.111004.

DOI: https://doi.org/10.1016/j.pscychresns.2019.111004

The article is under copyright by Elsevier, the publisher should be contacted for permission to reuse or reprint the material in any form.

\title{
Electrophysiological Correlates of Visual Backward Masking in Patients with Major Depressive Disorder
}

Ophélie Favrod ${ }^{\mathrm{a}}$, Janir R. da Cruz ${ }^{\mathrm{a}, \mathrm{b}}$, Maya Roinishvilii, ${ }^{\mathrm{c}, \text { Ekaterine Berdzenishvilie, Andreas }}$ Brand $^{\mathrm{a}}$, Patrícia Figueiredo $^{\mathrm{b}}$, Michael H. Herzog $^{\mathrm{a}}$, Eka Chkonia $^{\mathrm{d}, \mathrm{e}}$

\begin{abstract}
Depression and schizophrenia are two psychiatric diseases with high co-morbidity. For this reason, it is important to find sensitive endophenotypes, which may disentangle the two disorders. The Shine-Through paradigm, a visual backward masking task, is a potential endophenotype for schizophrenia. Masking is strongly deteriorated in schizophrenia patients, which is reflected in reduced EEG amplitudes. Here, we tested whether masking deficits and associated EEG changes are also found in patients with major depressive disorder. First, we replicated previous findings showing that depressive patients exhibit, at most, only weak masking deficits. Second, we found that the EEG amplitudes of depressive patients were reduced compared to controls and slightly increased compared to schizophrenia patients. As a secondary analysis, we compared the performance in the masking paradigm with three cognitive tasks, namely: the Wisconsin card sorting test, a verbal fluency test and a degraded continuous performance test. Performance in all but the verbal fluency test could discriminate schizophrenia from depression.
\end{abstract}




\section{Introduction}

Depression and schizophrenia are two major psychiatric diseases, which exhibit a considerable overlap in terms of their psychopathology (schizoaffective disorders) and their symptoms (negative symptoms: Andreasen, 1982; Dai et al., 2018; delusions: Sax et al., 1996). There is also a strong genetic overlap between the two disorders (Lee et al., 2013; Smoller et al., 2019; Postolache et al., 2019). It is therefore of great interest to disentangle the neural underpinnings of the two diseases. For this purpose, endophenotypes are of crucial importance. An endophenotype must be: associated with the disease, inheritable, present at a higher rate within relatives, and stateindependent (Gottesman and Gould, 2003).

Deficits in visual backward masking tasks, in particular the Shine-Through paradigm, have been proposed as potential endophenotypes of schizophrenia (Chkonia et al., 2010a). In the ShineThrough paradigm, a target vernier is followed by a grating mask. A vernier is composed of two vertical bars separated by a small horizontal gap, and the task is to report whether the lower bar is offset to the left or to the right. The mask deteriorates the visibility of the target vernier. We measured the Stimulus Onset Asynchrony (SOA) between the onset of the venier and the onset of the mask in an adaptive manner for each individual, such that $75 \%$ of correct responses were achieved. Schizophrenia patients needed SOAs of about 5 times longer than controls (Herzog et al., 2004), and healthy siblings of patients needed SOAs of about 2.5 longer than controls (Chkonia et al., 2010a). Masking was, not only impaired in schizophrenia patients, but also in schizoaffective and bipolar patients. On the other hand, patients with depression showed no deficit in visual masking (Chkonia et al., 2012). For this reason, the Shine-Through paradigm was considered to be an endophenotype of the schizophrenia spectrum (or psychosis spectrum).

Masking deficits of schizophrenia patients were reflected in the electroencephalogram (EEG), being strongly associated with a reduced N1 component of event-related potentials (ERPs) as measured by the global field power (GFP; Plomp et al., 2013; da Cruz et al., in revision). For patients with first-episode psychosis, the neural correlates were at an intermediate level: GFP N1 traces were similar to those of controls when the task was easy, and similar to chronic schizophrenia patients when the task was challenging (Favrod et al., 2018). Comparably to controls, observers with high scores of schizotypy showed reduced GFP N1 amplitudes (Favrod et al., 2017). Surprisingly, siblings of schizophrenia patients showed higher GFP N1 amplitudes than controls, suggesting a compensation mechanism (da Cruz et al., in revision).

As mentioned, schizophrenia patients were impaired in the Shine-Through paradigm whereas depressive patients were not (Chkonia et al., 2012). The aim of the present study was to investigate the electrophysiological correlates of masking in major depressive disorder. Although the electrophysiological correlates are not the endophenotype themselves, they help to understand its underlying mechanisms. We also compared the Shine-Through paradigm to other cognitive tasks that often show impairments in schizophrenia patients, namely: the degraded continuous performance test (CPT), the Wisconsin card sorting test (WCST), and a verbal fluency test (VFT). 


\section{Methods}

$\underline{\text { Visual Backward Masking }}$

Stimuli were displayed on a Siemens Fujitsu P796-1 monitor with a refresh rate of $100 \mathrm{~Hz}$. The screen resolution was $1024 \times 768$ pixels. Patients sat in a dimly illuminated room, at a distance of $3.5 \mathrm{~m}$ away from the monitor. The vernier and mask stimuli were presented in white (with a luminance of $\left.100 \mathrm{~cd} / \mathrm{m}^{2}\right)$ on a black background $\left(<1 \mathrm{~cd} / \mathrm{m}^{2}\right)$.

The vernier stimuli consisted of two vertical bars separated by a vertical gap of 1' (arc min). The lower bar was slightly offset either to the left or to the right compared to the upper one. The horizontal vernier offset was 1.2'. Following the vernier, a mask was presented which consisted of two rows of either five or twenty-five aligned bars. The horizontal spacing between mask elements was 3.33'. An illustration of the stimuli display can be found in the supplementary material (Figure S1). Observers reported the offset direction of the lower bar by pushing one of the two hand-held buttons.

\section{Adaptive experiment}

For each observer, we found the vernier duration (VD) for which the threshold of offset discrimination was below 0.6'. Afterwards, we used the individual VD with a fixed vernier offset of 1.2', and determined the stimulus onset asynchrony (SOA) threshold for each participant using an adaptive strategy (Parametric Estimation by Sequential Testing; Taylor, 1967) such that $75 \%$ correct responses were achieved. The procedure is described in detail in (Cappe et al., 2012; Herzog et al., 2004; Shaqiri et al., 2015, Favrod et al., 2018).

\section{EEG experiment}

For the EEG experiment, we used only the 25-element mask. As in previous EEG studies (Favrod et al., 2017; Favrod et al., 2018; Plomp et al., 2013), we tested four conditions: Vernier Only, Long SOA, Short SOA, and Mask Only. In the Vernier Only condition, the vernier was presented alone for $30 \mathrm{~ms}$, which corresponds to the mean VD of schizophrenia patients in previous studies. In the Short and Long SOA conditions, the vernier was presented for $30 \mathrm{~ms}$ followed by a mask for 300 ms with an SOA of either 30 or $150 \mathrm{~ms}$, which are the average SOAs for controls and schizophrenia patients, respectively. In the Mask Only condition, the mask was presented for $300 \mathrm{~ms}$; no Vernier was presented.

For each observer, 8 blocks of 80 trials (20 trials/condition) were presented. The conditions order was pseudo-randomized within each block. In total, there were 160 trials per condition.

\section{EEG recordings and pre-processing}

We used the EEG BioSemi Active Two system with $64 \mathrm{Ag}-\mathrm{AgCl}$ sintered active electrodes distributed across the scalp according to the 10/20 layout system. The sampling frequency was 
2048 Hz. EEG data were pre-processed offline in Matlab (R2012a, The MathWorks Inc., Natick, MA) with EEGLAB (Delorme and Makeig, 2004) and using an automated pre-processing pipeline (APP; da Cruz et al., 2018). The signal was down-sampled to $512 \mathrm{~Hz}$, band-passed filtered from 1 to $40 \mathrm{~Hz}$, and the $50 \mathrm{~Hz}$ line noise was removed using CleanLine (Mullen, 2012). Signal was rereferenced to the biweight estimate of the mean of all electrode-channels (Hoaglin et al., 1983, 2011). Unstable and noisy channels were removed and interpolated with a 3D spline function. Eye blink artifacts were removed through an independent component analysis (ICA) approach. EEG epochs were extracted from a time interval comprising $100 \mathrm{~ms}$ before the stimulus onset (baseline) and $400 \mathrm{~ms}$ after stimulus onset, i.e. [-100; +400] ms relative to stimulus onset, and these then rereferenced to the common average. Reaction times outside the interval [300;3000] ms were excluded. The EEG epochs corresponding to hits and missed trials were averaged together and baseline corrected, for each condition and each participant.

\section{EEG analysis}

The individual visual evoked potentials (VEP) were analyzed in MATLAB (R2018b, The MathWorks Inc., Natick, MA). Signal from two occipital electrodes (PO7 and PO8) were extracted to visualize the positive and negative components of the VEPs (supplementary material Figure S2). The Global Field Power (GFP) was computed as the standard deviation across all electrodes at each time point, for each participant and each condition separately (Lehmann and Skrandies, 1980). We selected this measure to avoid the problem of selecting an electrode arbitrarily and also potential reference problems. Grand averages of GFPs were computed for each condition, each session, and each group of participants.

\section{CPT, WCST, and VFT}

We administered a computerized version of the Nelson Test (Nelson, 1976), which is a modified Wisconsin Card Sorting Test (WCST, Berg, 1948) with 48 cards. We recorded the number of categories that participants went through, as well as the numbers of correct responses, errors and preservative errors. We also administered a degraded Continuous Performance Test (CPT, Rosvold et al., 1956). We report d' values. Methodological details can be found in Chkonia et al., 2010a. The Verbal Fluency Test (VFT) was derived from the Benton Controlled Oral Word Association Test (COWAT, Ruff et al., 1996). We asked the participants to report as many words as they could, belonging to either the animal or fruit/vegetables category. The number of words for each category was reported.

\section{$\underline{\text { Statistical analysis }}$}

Repeated measures ANOVAs were performed using JASP (https://jasp-stats.org/, version 0.9.1.0). Statistical tests were Greenhouse-Geisser corrected for lack of sphericity when necessary, and were Bonferroni-Holm corrected for multiple comparisons.

Participants 
All participants had normal or corrected-to-normal vision; with a visual acuity superior or equal to 0.8 determined for both eyes with FrAct (Bach, 1996). All participants signed an informed consent and were informed that they could quit the experiments at any time. All procedures complied with the Declaration of Helsinki and were approved by the local ethics committee.

General exclusion criteria were: drug or alcohol abuse, and neurological or other somatic illnesses influencing the subjects' mental state.

\section{Depressive patients}

Patients were recruited either from the Tbilisi Mental Health Hospital or from the Acute Psychiatric Departments of Multiprofile Clinics. Patients were diagnosed with major depressive affective disorder in a recurrent episode according to the Diagnostic and Statistical Manual of Mental Disorders IV/V, by means of an interview based on the Structured Clinical Interview, information of the staff, and the study of the records (mild: 296.31, $\mathrm{n}=14$; moderate: 296.32, $\mathrm{n}=18$; severe: 296.33, n=7). Psychopathology was assessed by an experienced psychiatrist (EC) through the Brief Psychiatric Rating Scale (BPRS; Overall and Gorham, 1962) and the Hamilton scale (Hamilton, 1960). No depressive patient had an additional axis I disorder.

In total, 39 depressive patients were recruited. Not all of them participated in each task for various reasons. Thirty-eight performed the WCST. Thirty-seven performed the CPT degraded test. Thirty-six performed the VFT. Thirty-four performed the masking task. Finally, twenty-six of them performed the masking paradigm with EEG. Group characteristics are depicted in Table 1 for the two masking experiments (behavioral / adaptive and EEG / fixed SOA) and in Table S1 in the supplementary material for the other data subset (CPT, WCST, and VF). Out of the 34 patients that performed the masking task, 30 patients took antidepressants (fluoxetine, fluvoxamine, clomipramine, venlafaxine, escitalopram) and 24 patients were receiving neuroleptic medication. Out of the 26 patients who performed the EEG, 24 patients received antidepressants and 19 were receiving neuroleptic medication. The depressive patients were recovering from depression and were tested when stabilized. At the time of testing, patients had Hamilton scores not higher than 28. The majority of the depressive patients were taking neuroleptic medication as an adjunctive treatment either as maintenance dose or as sedation. Patients not receiving traditional antidepressants were treated with quetiapine or aripiprazole as neuroleptics for antidepressant effects.

Table 1| Demographics for the controls, the depressive patients (two groups: the adaptive experiment and the EEG experiment subgroup) and schizophrenia patients

\begin{tabular}{ccccc}
\hline & Controls & $\begin{array}{c}\text { Depression } \\
\text { (adaptive) }\end{array}$ & $\begin{array}{c}\text { Depression } \\
\text { (EEG) }\end{array}$ & Schizophrenia \\
\hline $\boldsymbol{n}$ & 76 & 34 & 26 & 90 \\
\hline Gender (F/M) & $34 / 42$ & $22 / 12$ & $16 / 10$ & $15 / 75$ \\
\hline Age (years) \pm SD & $34.9 \pm 8.1$ & $32.6 \pm 10.0$ & $32.3 \pm 9.6$ & $35.8 \pm 8.8$ \\
\hline
\end{tabular}


Education (years) \pm SD

$15.0 \pm 2.8$

$14.8 \pm 2.6$

$14.8 \pm 2.6$

$13.2 \pm 2.7$

\begin{tabular}{|c|c|c|c|c|}
\hline $\begin{array}{l}\text { Illness Duration (months) } \pm \\
\text { SD }\end{array}$ & & $5.9 \pm 5.2$ & $6.2 \pm 5.5$ & $11.3 \pm 8.0$ \\
\hline SANS \pm SD & & & & $10.7 \pm 5.3$ \\
\hline SAPS \pm SD & & & & $10.0 \pm 8.1$ \\
\hline BPRS & & $31.4 \pm 6.9$ & $30.4 \pm 5.0$ & \\
\hline Hamilton & & $15.8 \pm 5.6$ & $14.9 \pm 5.4$ & \\
\hline $\mathrm{CPZ}$ equivalent $\pm \mathrm{SD}$ & & $236 \pm 246.4$ & $215 \pm 182.3$ & $549.3 \pm 398.5$ \\
\hline Handedness (L/R) & $5 / 71$ & $1 / 33$ & $1 / 25$ & $4 / 86$ \\
\hline Visual Acuity \pm SD & $1.6 \pm 0.4$ & $1.4 \pm 0.4$ & $1.4 \pm 0.4$ & $1.5 \pm 0.4$ \\
\hline
\end{tabular}

Note: Only 29 of the 34 depressive patients were assessed with the Hamilton scale (and 22 for the EEG dataset). 72 of the 90 schizophrenic patients received neuroleptic medication.

\section{Controls and schizophrenia patients}

The results obtained for the schizophrenia patients and controls are also reported in da Cruz et al., in revision. We used them here for comparison with the depressive group and re-plotted them. The EEG data for three groups were collected and analyzed using the same experimental setup and preprocessing pipeline, as described in a previous section (APP; da Cruz et al., 2018). Psychopathology was assessed by the Scales for the assessment of negative and positive symptoms (SANS, Andreasen 1984a; SAPS, Andreasen 1984b). Out of the 76 controls, 75 performed the CPT, 74 the WCST and 48 the VFT. Out of the 90 patients, 89 of them did the WCST, 88 the CPT and 23 the VFT. All of them were considered for the masking experiments (adaptive and EEG).

\section{Results}

$\underline{\text { Visual Backward Masking }}$

The VD was significantly lower for controls compared to both groups of patients, as can be seen in Figure 1a and Table 2.

(a) Thresholds in the adaptive experiment

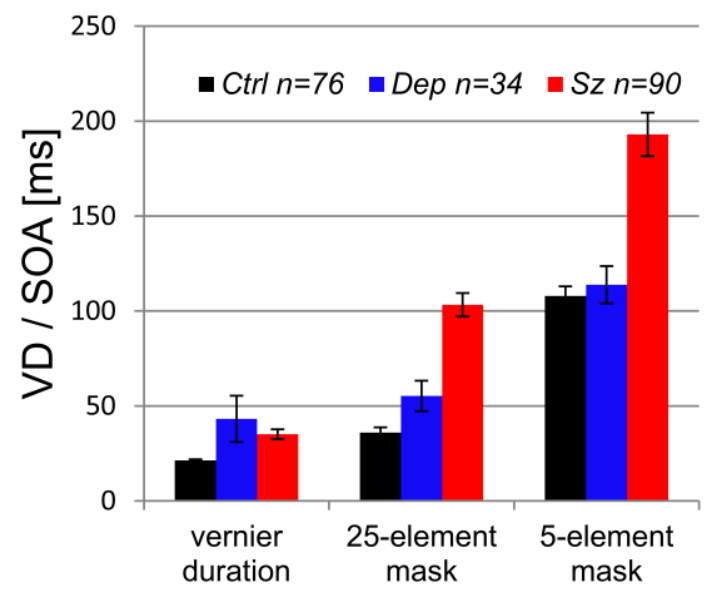

(b) Percent correct in the EEG experiment

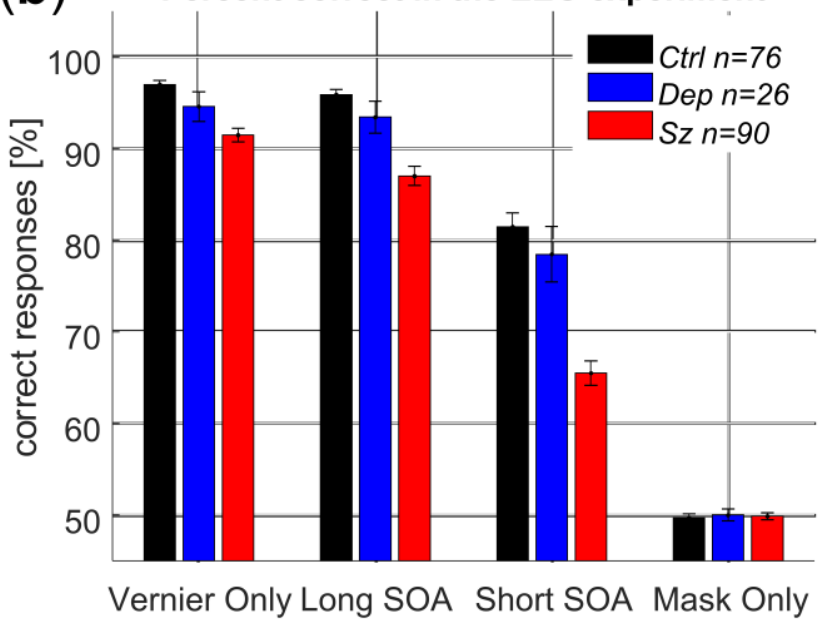


Figure 1| Behavioral performance (a) Thresholds obtained with the adaptive procedure, for controls (Ctrl), depressive patients (Dep) and schizophrenia patients (Sz): Vernier duration (VD) for the vernier condition and the Stimulus Onset Asynchronies (SOA) for the two masking conditions with the 5- and 25-element masks (b) Percentage of correct responses in each condition of the EEG experiment (25-element mask) for the Ctrl, Dep, and Sz groups. Depressive patients were almost at the same level as controls.

Schizophrenia patients performed significantly worse (longer SOAs) than both depressive patients and controls (Figure 1a, Table 2). There was no significant difference in SOAs between controls and depressive patients when both masks were combined (main effect, Table 2), replicating previous findings from Chkonia et al., 2012. When considering the interaction between the 5- and 25-element masks, one comparison turned out to be significant: depressive patients performed significantly ( $\mathrm{p}_{\text {holm }}=0.045$ ) worse than controls with the 25-element mask (Table S3 of the supplementary material). This result contradicted previous findings. In the EEG experiment (25element mask), we found no significant difference in performance between controls and depressive patients. Consistently, we did find significant differences between controls and schizophrenia patients and between depressive and schizophrenia patients (Table 2, Figure 1b). In summary, depressive patients performed better than schizophrenia patients and similarly to controls in most masking conditions (the 25 -element mask of the adaptive procedure being the only exception).

Table 2| Statistical analysis for the visual backward masking performance: adaptive masking experiment (Vernier, 5- and 25-element masks) and EEG experiment.

\begin{tabular}{|c|c|c|c|c|c|c|}
\hline \multicolumn{6}{|c|}{ Vernier Duration } & Comments \\
\hline group & \multicolumn{5}{|c|}{$F(2,197)=6.08, p=0.003, \eta^{2}=0.058$} & \\
\hline post-hoc & \multicolumn{2}{|c|}{ Ctrl vs Dep } & \multicolumn{2}{|r|}{ Ctrl vs Sz } & Dep vs Sz & \\
\hline & \multicolumn{2}{|c|}{$\begin{array}{c}t(108)=-3.161, \\
\mathrm{~d}=-0.553, \\
p_{\text {holm }}=0.005\end{array}$} & \multicolumn{2}{|c|}{$\begin{array}{c}t(164)=-2.635, \\
\mathrm{~d}=-0.753, \\
p_{\text {holm }}=0.018\end{array}$} & $\begin{array}{c}t(122)=-1.201, \\
\mathrm{~d}=0.191, \\
p_{\text {holm }}=0.231\end{array}$ & \\
\hline \multicolumn{6}{|c|}{ 5-element and 25-element masks (SOA) } & \\
\hline \multicolumn{2}{|c|}{ mask } & \multicolumn{4}{|c|}{$F(1,197)=230.420, p<0.001, \eta^{2}=0.530$} & \\
\hline \multicolumn{2}{|c|}{ SOA5 vs SOA25 } & \multicolumn{4}{|c|}{$t(197)=17.32, \mathrm{~d}=1.225, p_{\text {holm }}<0.001$} & \\
\hline \multicolumn{2}{|c|}{ mask*group } & \multicolumn{4}{|c|}{$F(2,197)=3.570, p=0.030, \eta^{2}=0.016$} & $\begin{array}{l}\text { simple main effects and } \\
\text { post-hoc statistics are } \\
\text { reported in the } \\
\text { supplementary material } \\
\text { (Table S3) }\end{array}$ \\
\hline \multicolumn{2}{|c|}{ group } & \multicolumn{4}{|c|}{$F(2,197)=38.39, p<0.001, \eta^{2}=0.280$} & \\
\hline \multirow{2}{*}{\multicolumn{2}{|c|}{ post-hoc }} & \multicolumn{2}{|c|}{ Ctrl vs Dep } & Ctrl vs Sz & Dep vs Sz & \\
\hline & & \multicolumn{2}{|c|}{$\begin{array}{c}t(218)=-1.045, \\
\mathrm{~d}=-0.074, \\
p_{\text {holm }}=0.297\end{array}$} & $\begin{array}{c}t(330)=-8.366 \\
\mathrm{~d}=-0.592 \\
p_{\text {holm }}<0.001\end{array}$ & $\begin{array}{c}t(246)=-5.403, \\
\mathrm{~d}=-0.382, \\
p_{\text {holm }}<0.001\end{array}$ & \\
\hline
\end{tabular}




\begin{tabular}{|c|c|c|c|c|}
\hline \multicolumn{4}{|c|}{ Percent correct in the EEG experiment } & \multirow{3}{*}{$\begin{array}{l}\text { simple main effects and } \\
\text { post-hoc statistics are } \\
\text { reported in the } \\
\text { supplementary material } \\
\text { (Table S3) }\end{array}$} \\
\hline condition & \multicolumn{3}{|c|}{$F(1.788,338.005)=1285.76, p<0.001, \eta^{2}=0.847$} & \\
\hline condition*group & \multicolumn{3}{|c|}{$F(3.577,338.005)=21.40, p<0.001, \eta^{2}=0.028$} & \\
\hline group & \multicolumn{3}{|c|}{$F(2,189)=31.71, p<0.001, \eta^{2}=0.251$} & \\
\hline post-hoc & Ctrl vs Dep & Ctrl vs Sz & Dep vs Sz & \\
\hline & $\begin{array}{c}t(406)=1.334 \\
\mathrm{~d}=0.096 \\
\text { pholm }=0.184\end{array}$ & $\begin{array}{c}t(662)=7.785, \\
\mathrm{~d}=0.562, \\
p_{\text {holm }}<0.001\end{array}$ & $\begin{array}{c}t(462)=4.085, \\
\mathrm{~d}=0.295, \\
p_{\text {holm }}<0.001\end{array}$ & \\
\hline
\end{tabular}

Visual Backward Masking: Global Field Power

We performed the repeated measures ANOVA for the peak of the GFP grand average (N1 component), for each group and each condition, as shown in Figure 2 (peaks and latencies are shown in Table S2, in the supplementary material). 

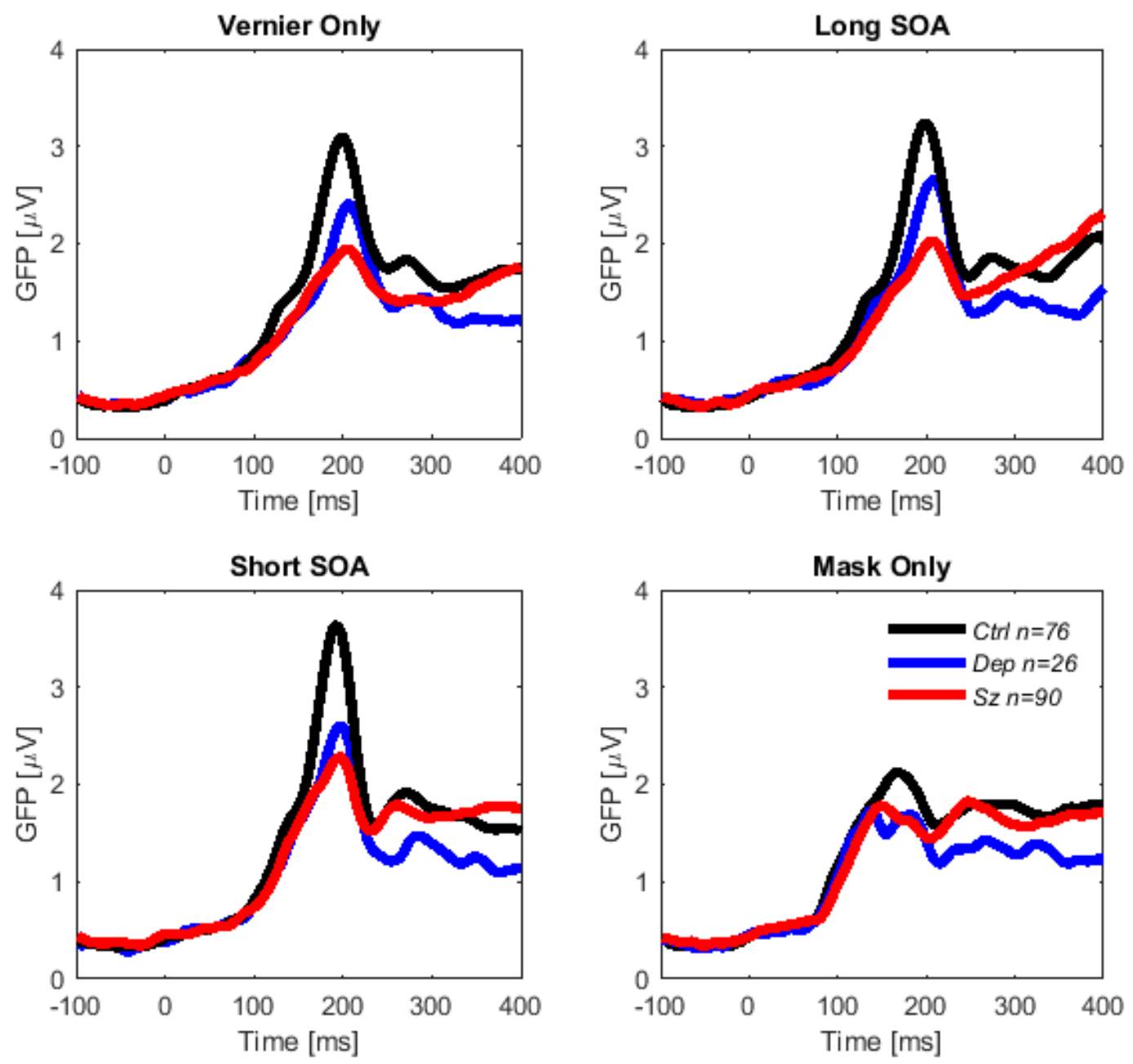

Figure 2| Global Field Power (GFP) for the four EEG conditions with the 25-element mask. Black: controls (Ctrl); Blue: depressive patients (Dep), and Red: schizophrenia patients (Sz). In all four conditions with a vernier, the N1 component peaks around $200 \mathrm{~ms}$.

We found a strong and significant difference between controls and schizophrenia patients, a significant difference between controls and depressive patients and no significant difference between depressive and schizophrenia patients. In particular, compared to schizophrenia patients, patients with depression showed a significantly greater GFP N1 amplitude in the Long SOA condition and a marginally greater GFP N1 amplitude in the Vernier Only condition. There was no difference across groups in the Mask Only condition. Statistics are reported in Table 3.

For comparative analysis with previous studies (Favrod et al., 2017; 2018; da Cruz et al., in revision), statistics across the whole epoch (i.e., computed at each time frame) are shown in the supplementary material (Figure S3). 
In summary, the GFP N1 amplitudes of depressive patients were reduced compared to controls and increased compared to schizophrenia patients (in particular for the Long SOA condition and only slightly for the Vernier Only condition).

Table 3| Statistical analysis for the GFP N1 peak measured in the EEG experiment.

\begin{tabular}{|c|c|c|c|c|}
\hline \multicolumn{4}{|c|}{ N1 peak $(\sim 200 \mathrm{~ms})$} & \multirow[t]{2}{*}{ Comments } \\
\hline group & \multicolumn{3}{|c|}{$F(2,189)=20.26, p<0.001, \eta^{2}=0.177$} & \\
\hline post-hoc & Ctrl vs Dep & Ctrl vs Sz & Dep vs Sz & \\
\hline & $\begin{array}{c}t(406)=2.883 \\
\mathrm{~d}=0.208 \\
p_{\text {holm }}=0.009\end{array}$ & $\begin{array}{c}t(662)=6.341 \\
\mathrm{~d}=0.458 \\
p_{\text {holm }}<0.001\end{array}$ & $\begin{array}{c}t(462)=1.495 \\
\mathrm{~d}=0.108 \\
p_{\text {holm }}=0.137\end{array}$ & \\
\hline condition & \multicolumn{3}{|c|}{$F(2.108,398.337)=60.01, p<0.001, \eta^{2}=0.221$} & $\begin{array}{c}\text { post-hoc statistics are } \\
\text { shown in the } \\
\text { supplementary } \\
\text { material (Table S3) }\end{array}$ \\
\hline condition*group & \multicolumn{3}{|c|}{$F(4.215,398.337)=11.32, p<0.001, \eta^{2}=0.083$} & \\
\hline post-hoc & Ctrl vs Dep & $\begin{array}{c}\text { Ctrl vs Sz } \\
\text { (da Cruz et al., } \\
\text { submitted) }\end{array}$ & Dep vs Sz & \\
\hline Vernier Only & $\begin{array}{c}t(100)=2.564 \\
\mathrm{~d}=0.539 \\
p_{\text {holm }}=0.022\end{array}$ & $\begin{array}{c}t(164)=6.279 \\
\mathrm{~d}=0.951 \\
p_{\text {holm }}<0.001\end{array}$ & $\begin{array}{c}t(114)=1.777 \\
\mathrm{~d}=0.451 \\
\text { pholm }=0.077\end{array}$ & $\begin{array}{c}F(2,189)=19.74, \\
p<0.001, \eta^{2}=0.173\end{array}$ \\
\hline Long SOA & $\begin{array}{c}t(100)=2.195 \\
\mathrm{~d}=0.457 \\
p_{\text {holm }}=0.037\end{array}$ & $\begin{array}{c}t(164)=6.599, \\
\mathrm{~d}=1.011 \\
p_{\text {holm }}<0.001\end{array}$ & $\begin{array}{c}t(114)=2.377, \mathrm{~d} \\
=0.598 \\
p_{\text {holm }}=0.037\end{array}$ & $\begin{array}{c}F(2,189)=21.81, \\
p<0.001, \eta^{2}=0.188\end{array}$ \\
\hline Short SOA & $\begin{array}{c}t(100)=3.330 \\
\mathrm{~d}=0.685 \\
p_{\text {holm }}=0.002\end{array}$ & $\begin{array}{c}t(164)=6.378, \\
\mathrm{~d}=0.967, \\
p_{h o l m}<0.001\end{array}$ & $\begin{array}{c}t(114)=1.065 \\
\mathrm{~d}=0.278 \\
p_{\text {holm }}=0.288\end{array}$ & $\begin{array}{c}F(2,189)=20.87, \\
p<0.001, \eta^{2}=0.181\end{array}$ \\
\hline Mask Only & $\begin{array}{c}t(100)=1.885 \\
\mathrm{~d}=0.416 \\
p_{\text {holm }}=0.122\end{array}$ & $\begin{array}{c}t(164)=2.419 \\
\mathrm{~d}=0.367 \\
p_{\text {holm }}=0.050\end{array}$ & $\begin{array}{c}t(114)=-0.231 \\
\mathrm{~d}=-0.055 \\
p_{\text {holm }}=0.817\end{array}$ & $\begin{array}{c}F(2,189)=3.489 \\
p=0.032, \eta^{2}=0.036\end{array}$ \\
\hline
\end{tabular}

\section{CPT, VFT, and WCST}

For the CPT, we found a group difference between controls and schizophrenia patients as shown by the post-hoc tests (Table 4). Depressive patients were also significantly different from schizophrenia patients (Figure 3a). 

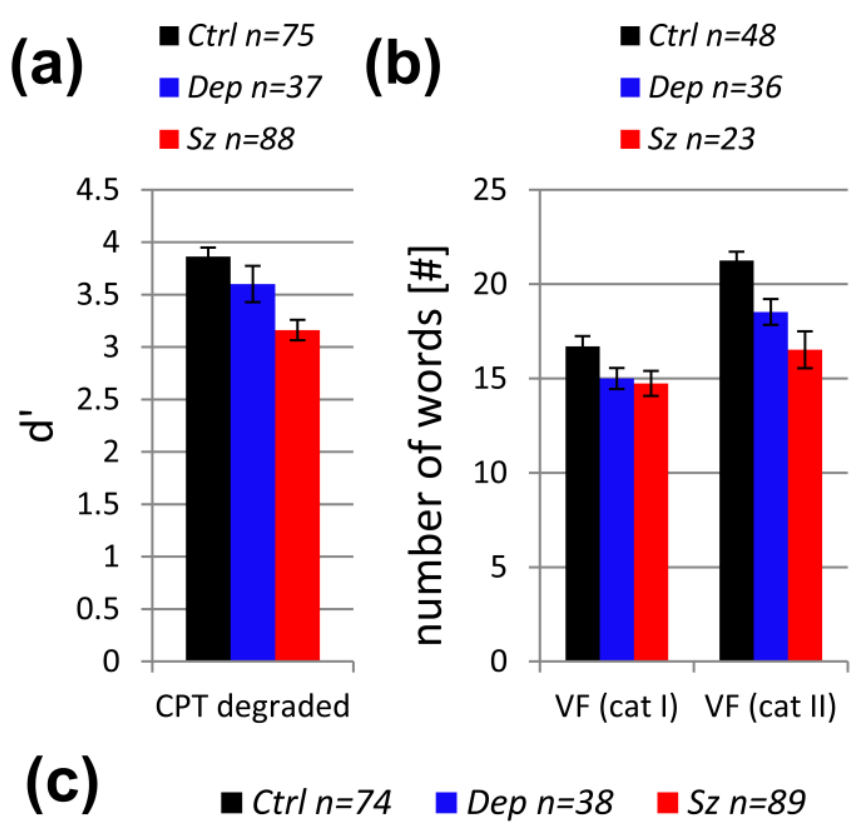

Dep $n=38 \quad$ Sz $n=89$

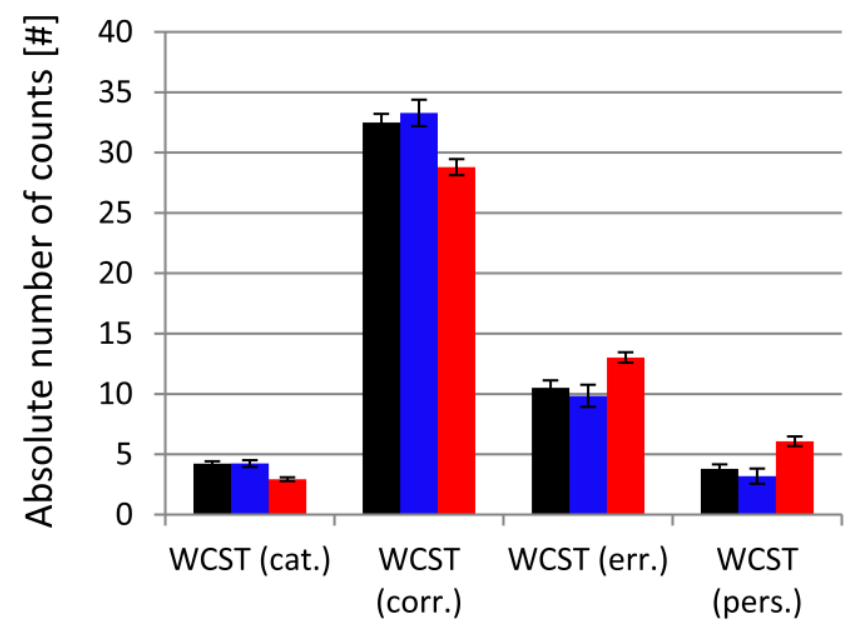

Figure 3| Performance for the Continuous Performance Test (CPT), the Verbal Fluency Test (VFT) and the Wisconsin Card Sorting Test (WCST), in the three groups (Ctrl, Dep and Sz). (a) d' for the CPT (b) number of words for the VFT - category I: animals, category II: vegetables/fruits (c) the four different measures for the WCST: the categories, the number of correct responses, the number of errors and the perseverative errors.

In the VFT, we found a group effect, with controls performing better than schizophrenia patients and also better than depressive patients (Table 4). No significant differences were found between depressive and schizophrenia patients (Figure 3b), which spoke against VFT as an endophenotype for schizophrenia because of the lack of specificity. 
For the WCST, we found a significant interaction between measure and group, showing that schizophrenia patients made more mistakes, were less accurate and were going through fewer categories compared to controls and depressive patients (Figure 3c). We did not find any significant differences between controls and depressive patients for either measure. Statistical tests are reported in Table 4.

In summary, we found significant differences between controls and schizophrenia patients for all tasks. We found a significant difference between controls and depressive patients for VD, the GFP N1 amplitude and the VFT. Finally, we found significant differences between depressive and schizophrenia patients for the SOAs (adaptive masking), the CPT and the WCST.

Table 4| Statistical analysis for the three cognitive tasks: degraded continuous performance test $(C P T)$, verbal fluency test (VFT), and Wisconsin card sorting test (WCST).

\begin{tabular}{|c|c|c|c|c|}
\hline \multicolumn{4}{|c|}{ CPT } & Comments \\
\hline group & \multicolumn{3}{|c|}{$F(2,197)=13.32, p<0.001, \eta^{2}=0.119$} & \\
\hline \multirow[t]{2}{*}{ post-hoc } & Ctrl vs Dep & Ctrl vs Sz & Dep vs Sz & \\
\hline & $\begin{array}{c}t(110)=1.499, \\
\mathrm{~d}=0.311, \\
p_{\text {holm }}=0.136\end{array}$ & $\begin{array}{c}t(161)=5.109, \\
\mathrm{~d}=0.846, \\
p_{\text {holm }}<0.001\end{array}$ & $\begin{array}{c}t(123)=2.561, \\
\mathrm{~d}=0.461, \\
p_{\text {holm }}=0.022\end{array}$ & \\
\hline \multicolumn{4}{|c|}{ VFT } & \\
\hline group & \multicolumn{3}{|c|}{$F(2,104)=10.48, p<0.001, \eta^{2}=0.168$} & \\
\hline \multirow[t]{2}{*}{ post-hoc } & Ctrl vs Dep & Ctrl vs Sz & Dep vs Sz & \\
\hline & $\begin{array}{c}t(160)=3.213, \\
\mathrm{~d}=0.311, \\
p_{\text {holm }}=0.003\end{array}$ & $\begin{array}{c}t(140)=4.223, \\
\mathrm{~d}=0.408, \\
p_{\text {holm }}<0.001\end{array}$ & $\begin{array}{c}t(110)=1.358, \\
\mathrm{~d}=0.131 \\
p_{\text {holm }}=0.177\end{array}$ & \\
\hline category & \multicolumn{3}{|c|}{$F(1,104)=66.644, p<0.001, \eta^{2}=0.374$} & \multirow{2}{*}{$\begin{array}{l}\text { simple main } \\
\text { effects and post- } \\
\text { hoc tests are } \\
\text { reported in the } \\
\text { supplementary } \\
\text { material (Table } \\
\text { S3) }\end{array}$} \\
\hline category*group & \multicolumn{3}{|c|}{$F(2,104)=3.753, p=0.027, \eta^{2}=0.042$} & \\
\hline \multicolumn{4}{|c|}{ WCST } & \\
\hline group & \multicolumn{3}{|c|}{$F(2,198)=0.631, p=0.533, \eta^{2}=0.015$} & \\
\hline measure & \multicolumn{3}{|c|}{$F(1.304,258.141)=1156.75, p<0.001, \eta^{2}=0.841$} & $\begin{array}{l}\text { post-hoc tests } \\
\text { are found in the } \\
\text { supplementary } \\
\text { material (Table } \\
\text { S3) }\end{array}$ \\
\hline measure*group & \multicolumn{3}{|c|}{$F(2.607,258.141)=10.24, p<0.001, \eta^{2}=0.015$} & $\begin{array}{l}\text { all simple main } \\
\text { effects were } \\
\text { significant } \\
\text { p }<0.001\end{array}$ \\
\hline post-hoc & Ctrl vs Dep & Ctrl vs Sz & Dep vs Sz & \\
\hline
\end{tabular}




\begin{tabular}{|c|c|c|c|c|}
\hline \multirow{3}{*}{ category } & $t(110)=-0.023$, & $t(161)=5.222$, & $t(125)=4.263$, & \\
& $\mathrm{d}=-0.004$, & $\mathrm{d}=0.839$, & $\mathrm{d}=0.828$ & \\
& $p_{\text {holm }}=0.982$ & $p_{\text {holm }}<0.001$ & $p_{\text {holm }}<0.001$ & \\
\hline \multirow{3}{*}{ correct response } & $t(110)=-0.627$, & $t(161)=3.730$, & $t(125)=3.674$, & \\
& $\mathrm{d}=-0.125$, & $\mathrm{d}=0.597$, & $\mathrm{d}=0.699$, & \\
& $p_{\text {holm }}=0.531$ & $p_{\text {holm }}<0.001$ & $p_{\text {holm }}<0.001$ & \\
\hline \multirow{3}{*}{ error } & $t(110)=0.699$, & $t(161)=-3.314$, & $t(125)=-3.410$, & \\
& $\mathrm{d}=0.126$, & $\mathrm{d}=-0.544$ & $\mathrm{~d}=-0.696$, & \\
\hline \multirow{2}{*}{ perseverative } & $p_{\text {holm }}=0.485$ & $p_{\text {holm }}=0.002$ & $p_{\text {holm }}=0.002$ & \\
error & $t(110)=0.858$, & $t(161)=-4.050$, & $t(125)=-4.172$, & \\
& $\mathrm{d}=0.180$ & $\mathrm{~d}=-0.652$, & $\mathrm{d}=-0.757$, & \\
& $p_{\text {holm }}=0.392$ & $p_{\text {holm }}<0.001$ & $p_{\text {holm }}<0.001$ & \\
\hline
\end{tabular}

\section{Discussion}

In a series of previous publications, we have shown that the Shine-through masking paradigm is a potential endophenotype of schizophrenia. Differences were rather small for the unmasked vernier performance; however, patients needed much longer SOAs than healthy controls when the mask was presented, which makes the paradigm both spatially and temporally challenging. In the masking conditions, SOAs were 5 and 2 times longer in the 25- and 5-element mask conditions, respectively. Similar results were found for bipolar and schizoaffective patients (Chkonia et al., 2012). An important criterion for an endophenotype is that unaffected siblings of schizophrenia patients show deficits compared to controls. Indeed, we found that unaffected siblings show masking deficits compared to healthy controls (factor 2.5). The same was true for first episode psychosis patients and adolescents with psychosis (Favrod et al., 2018; Holzer et al., 2009). We did not find masking differences in depressive patients and abstinent alcoholics compared to controls (Chkonia et al., 2012). Hence, the Shine-through paradigm was specific for the psychosis spectrum. These results were surprising since depression is also a severe brain disease with often heavy medication. In addition, there is a substantial overlap between depression and schizophrenia in terms of both symptoms and genetics.

Here, we tested a new cohort of depressive patients and replicated previous masking behavior results. In the adaptive condition (5-element mask) and the fixed SOA condition (in the EEG experiment) with the 25-element mask, depressive patients performed approximately at the level of controls. In the adaptive condition with the 25-element mask, we found a significant difference in patients, contrary to previous results. One reason for this difference might be due to undersampling. Potentially, there was a very small difference between controls and depressive patients, which sometimes leads to significant results but mostly does not. Another plausible explanation emerged from a post-hoc analysis. We found that the difference between controls and depressive patients in the 25-element mask disappears when we control for gender (supplementary material Table S4-S7). There were more female participants in the depressive group than the schizophrenia group, and female participants perform slightly worse than male participants (Shaqiri et al., 2018). 
Hence, the small significant difference might be explained by a gender unbalance between the two samples.

Moreover, we also analyzed EEG data from the new cohort of depressive patients. In the Mask Only condition (when there was no task relevance), only a small N1 amplitude was elicited at around $200 \mathrm{~ms}$ after stimulus onset. Controls and schizophrenia patients showed no significant group differences. Hence, in schizophrenia patients, processing was mainly intact when no taskrelevant stimulus (target) was presented. However, when the target vernier was presented (Vernier Only, Long SOA, and Short SOA conditions), controls showed strongly increased N1 amplitudes, while these remained similar to the ones in the Mask Only condition for schizophrenia patients. We suggested that schizophrenia patients could not enhance the neural responses to the target vernier, making it more prone to masking (target enhancement deficit; Herzog and Brand, 2015). Importantly, the N1 amplitudes, even though moderately correlated with performance, do not reflect performance per se. In fact, in the Vernier Only condition, performance was similar between schizophrenia patients and controls (>90\%), but the ERP responses were different. We proposed then that the $\mathrm{N} 1$ amplitudes reflect, among other things, the amplification of the target (Herzog et al., 2013). Under normal everyday conditions, vernier-like differences go unnoticed. Only when the vernier is important, e.g. task-relevant, does the human brain enhance vernierrelated information by attention and neuromodulation. The cholinergic system may play an important role in this process (Bakanidze et al., 2013). We proposed that such target enhancement is deficient in schizophrenia patients, which is mainly evident in the demanding masking conditions. In support of this hypothesis, we found that, if healthy observers passively attended to task-irrelevant verniers, the verniers elicited only low N1 amplitudes, similar to those of schizophrenia patients (da Cruz, 2019; da Cruz et al., 2019). Interestingly, unaffected siblings of schizophrenia patients showed moderate performance deficits but increased N1 amplitudes compared to controls (da Cruz et al., in revision). We interpreted this increased N1 amplitudes in siblings as a compensatory mechanism, which would over-activate a network of brain regions. This over-activation, reflected in the N1 amplitudes, suggested that siblings engaged more effort in order to be able to perform better than patients.

Here, we found that depressive patients had good behavioral performance but relatively low N1 amplitudes. We, therefore, speculate that the $\mathrm{N} 1$ amplitudes reflect an interaction between target enhancement effects and how much intrinsic effort is put in the task. Potentially, depressive patients had no impairments regarding the stability of the representation (target enhancement), but they were disengaged in general, which was reflected in the lower EEG amplitudes. The depressive patients, however, complied with the task explaining the good performance.

An alternative explanation for the reduced $\mathrm{N} 1$ amplitudes in depression is that the neural correlates of masking are state-dependent (for depression). For example, N1 amplitudes might change depending on whether the depressive patients are in remission or in relapse, as evident for the P300 component (see Bruder et al., 2012, for a review). 
In all our studies on schizophrenia, we regularly conducted the CPT, VFT, and WCST. For this reason, we applied these tests also to depressive patients. For the VFT, depressive patients were impaired as much as schizophrenia patients. In contrast, in the CPT and WCST (number of errors), depressive patients performed better than schizophrenia patients, and were in fact almost at the level of controls. We would like to mention that the CPT is often proposed as an endophenotype for schizophrenia. However, results are mixed and evidence speaks rather against this claim because siblings of schizophrenia patients show no deficits (Chkonia et al., 2010b).

In summary, schizophrenia patients had a low ability to enhance faint targets, such as briefly presented verniers, which made them more prone to masking effects. Similarly, siblings of schizophrenia patients had low target enhancement compared to control. However, by engaging more resources they could partially compensate for the deficits, which improved performance compared to schizophrenia patients. However, this compensation was not sufficient to achieve healthy controls-like performance. In contrast to schizophrenia patients, depressive patients enhanced targets similarly to controls, and their performance was comparable to that of controls. However, depressive patients tended to perceive any kind of task as more difficult (Brinkmann \& Gendolla, 2008) and presumably disengaged from the task, which was reflected in their lower GFP N1 amplitudes compared to controls.

\section{Acknowledgements}

We would like to thank both anonymous reviewers for their comments, which greatly improved the manuscript. And we would like to thank Dr. Nicolas Silvestrini for the insightful discussion on depression and effort.

\section{Funding}

This work was supported by an NCCR Synapsy grant from the Swiss National Science Foundation (51NF40-185897), by the Portuguese Fundação para a Ciência e a Tecnologia, grant \# FCT $\mathrm{PD} / \mathrm{BD} / 105785 / 2014$ and by the "Knowledge Foundation" of Georgia. The authors have declared that there are no conflicts of interest in relation to the subject of this study.

\section{References}

Andreasen, N.C. and Olsen, S., 1982. Negative v positive schizophrenia: Definition and validation. Arch. of gen. psy., 39(7), pp.789-794.

Andreasen, N.C., 1984a. The scale for the assessment of negative symptoms (SANS). Rin. Seis. Iga., 13, pp.999-1010.

Andreasen, N.C., 1984b. The scale for the assessment of positive symptoms (SAPS). Uni. of Iowa, Iowa city.

Bach, M., 1996. The Freiburg Visual Acuity Test-automatic measurement of visual acuity. Opt.and vis. sci., 73(1), pp.49-53. 
Bakanidze, G., Roinishvili, M., Chkonia, E., Kitzrow, W., Richter, S., Neumann, K., Herzog, M., Brand, A. and Puls, I., 2013. Association of the nicotinic receptor $\alpha 7$ subunit gene (CHRNA7) with schizophrenia and visual backward masking. Front. in Psy., 4, p.133.

Berg, E.A., 1948. A simple objective technique for measuring flexibility in thinking. The jour. of gen.psych., 39(1), pp.15-22.

Brinkmann, K. and Gendolla, G.H., 2008. Does depression interfere with effort mobilization? Effects of dysphoria and task difficulty on cardiovascular response. Jour. of pers. and soc. psych., 94(1), p.146.

Bruder, G.E., Kayser, J. and Tenke, C.E., 2012. Event-related brain potentials in depression: clinical, cognitive and neurophysiologic implications. The Oxf. handbook of event-related potential components, 2012, pp.563-592.

Cappe, C., Herzog, M.H., Herzig, D.A., Brand, A. and Mohr, C., 2012. Cognitive disorganisation in schizotypy is associated with deterioration in visual backward masking. Psy. Res., 200(2-3), pp.652-659.

Chkonia, E., Roinishvili, M., Makhatadze, N., Tsverava, L., Stroux, A., Neumann, K., Herzog, M.H. and Brand, A., 2010. The shine-through masking paradigm is a potential endophenotype of schizophrenia. PLoS One, 5(12), p.e14268.

Chkonia, E., Roinishvili, M., Herzog, M.H. and Brand, A., 2010. First-order relatives of schizophrenic patients are not impaired in the Continuous Performance Test. Jour. of clin. and expe. neuropsy., 32(5), pp.481-486.

Chkonia, E., Roinishvili, M., Reichard, L., Wurch, W., Puhlmann, H., Grimsen, C., Herzog, M.H. and Brand, A., 2012. Patients with functional psychoses show similar visual backward masking deficits. Psy. Res., 198(2), pp.235-240.

da Cruz, J.R., Chicherov, V., Herzog, M.H. and Figueiredo, P., 2018. An automatic pre-processing pipeline for EEG analysis (APP) based on robust statistics. Clin. Neurophy., 129(7), pp.14271437.

da Cruz, J.R., 2019. EEG signatures of schizophrenia: visual backward masking and microstates (No. THESIS). EPFL.

da Cruz, J.R., Favrod, O., Johnston, P.R., Figueiredo, P., and Herzog, M.H. 2019. Neural correlates of target enhancement. Vis. Sci. Soc. (VSS) Annual Meeting, St-Pete Beach, FL, USA

da Cruz J.R.,Shaqiri A.,Roinishvili M.,Chkonia E.,Brand A.,Figueiredo P.,Herzog, M.H., in revision. Neural compensation mechanisms of siblings of schizophrenia patients as revealed by high-density EEG. Schiz. Bull. 
Dai, J., Du, X., Yin, G., Zhang, Y., Xia, H., Li, X., Cassidy, R., Tong, Q., Chen, D., Teixeira, A.L. and Zheng, Y., 2018. Prevalence, demographic and clinical features of comorbid depressive symptoms in drug naive patients with schizophrenia presenting with first episode psychosis. Schiz. Res., 193, pp.182-187.

Delorme, A. and Makeig, S., 2004. EEGLAB: an open source toolbox for analysis of single-trial EEG dynamics including independent component analysis. Jour. of neurosci. meth., 134(1), pp.921.

Favrod, O., Sierro, G., Roinishvili, M., Chkonia, E., Mohr, C., Herzog, M.H. and Cappe, C., 2017. Electrophysiological correlates of visual backward masking in high schizotypic personality traits participants. Psy. Res., 254, pp.251-257.

Favrod, O., Roinishvili, M., da Cruz, J.R., Brand, A., Okruashvili, M., Gamkrelidze, T., Figueiredo, P., Herzog, M.H., Chkonia, E. and Shaqiri, A., 2018. Electrophysiological correlates of visual backward masking in patients with first episode psychosis. Psy. Res.: Neuroi., 282, pp.64-72.

Gottesman, I.I. and Gould, T.D., 2003. The endophenotype concept in psychiatry: etymology and strategic intentions. Am. Jour. of Psy., 160(4), pp.636-645.

Hamilton, M., 1960. A rating scale for depression. Jour. of neurol., neurosur., and psy., 23(1), p.56.

Herzog, M.H., Kopmann, S. and Brand, A., 2004. Intact figure-ground segmentation in schizophrenia. Psy. res., 129(1), pp.55-63.

Herzog, M.H., Roinishvili, M., Chkonia, E. and Brand, A., 2013. Schizophrenia and visual backward masking: a general deficit of target enhancement. Front. in psych.y, 4, p.254.

Herzog, M.H. and Brand, A., 2015. Visual masking \& schizophrenia. Schiz. Res.: Cogn., 2(2), pp.64-71.

Hoaglin, D.C., Mosteller, F. and Tukey, J.W., 2000. Understanding robust and exploratory data analysis (No. Sirsi) i9780471384915).

Hoaglin, D.C., Mosteller, F. and Tukey, J.W. eds., 1985. Exploring data tables, trends, and shapes (Vol. 62). New York et al.: Wiley.

Holzer, L., Jaugey, L., Chinet, L. and Herzog, M.H., 2009. Deteriorated visual backward masking in the shine-through effect in adolescents with psychosis. Jour. of clin. and exp. neuropsy., 31(6), pp.641-647.

Lee, S.H., Ripke, S., Neale, B.M., Faraone, S.V., Purcell, S.M., Perlis, R.H., Mowry, B.J., Thapar, A., Goddard, M.E., Witte, J.S. and Absher, D., 2013. Genetic relationship between five psychiatric disorders estimated from genome-wide SNPs. Nat.gene., 45(9), p.984. 
Lehmann, D. and Skrandies, W., 1980. Reference-free identification of components of checkerboard-evoked multichannel potential fields. Electro. and clin. Neurophysi., 48(6), pp.609621.

Mullen, T., CleanLine. NITRC. 2012. https://www.nitrc.org/projects/cleanline/

Nelson, H.E., 1976. A modified card sorting test sensitive to frontal lobe defects. Cort., 12(4), pp.313-324.

Plomp, G., Roinishvili, M., Chkonia, E., Kapanadze, G., Kereselidze, M., Brand, A. and Herzog, M.H., 2012. Electrophysiological evidence for ventral stream deficits in schizophrenia patients. Schiz.bull., 39(3), pp.547-554.

Postolache, T.T., del Bosque - Plata, L., Jabbour, S., Vergare, M., Wu, R. and Gragnoli, C., 2019. Co - shared genetics and possible risk gene pathway partially explain the comorbidity of schizophrenia, major depressive disorder, type 2 diabetes, and metabolic syndrome. Am. Jour. of Med. Gen. Part B: Neuropsy. Gen., 180(3), pp.186-203.

Rosvold, H.E., Mirsky, A.F., Sarason, I., Bransome Jr, E.D. and Beck, L.H., 1956. A continuous performance test of brain damage. Jour. of cons. Psych., 20(5), p.343.

Ruff, R.M., Light, R.H., Parker, S.B. and Levin, H.S., 1996. Benton controlled oral word association test: Reliability and updated norms. Arch. of clin. Neuropsy., 11(4), pp.329-338.

Sax, K.W., Strakowski, S.M., Keck, P.E., Upadhyaya, V.H., West, S.A. and McElroy, S.L., 1996. Relationships among negative, positive, and depressive symptoms in schizophrenia and psychotic depression. The Brit. Jour. of Psy., 168(1), pp.68-71.

Shaqiri, A., Willemin, J., Sierro, G., Roinishvili, M., Iannantuoni, L., Rürup, L., Chkonia, E., Herzog, M.H. and Mohr, C., 2015. Does chronic nicotine consumption influence visual backward masking in schizophrenia and schizotypy?. Schiz. Res.: Cogn., 2(2), pp.93-99.

Shaqiri, A., Roinishvili, M., Grzeczkowski, L., Chkonia, E., Pilz, K., Mohr, C., Brand, A., Kunchulia, M. and Herzog, M.H., 2018. Sex-related differences in vision are heterogeneous. Sci. rep., 8(1), p.7521.

Smoller, J.W., Andreassen, O.A., Edenberg, H.J., Faraone, S.V., Glatt, S.J. and Kendler, K.S., 2019. Psychiatric genetics and the structure of psychopathology. Mol. Psy., 24(3), p.409.

Taylor, M. and Creelman, C.D., 1967. PEST: Efficient estimates on probability functions. The Jour. of the Acou. Soc. of Am., 41(4A), pp.782-787. 


\section{Supplementary material}

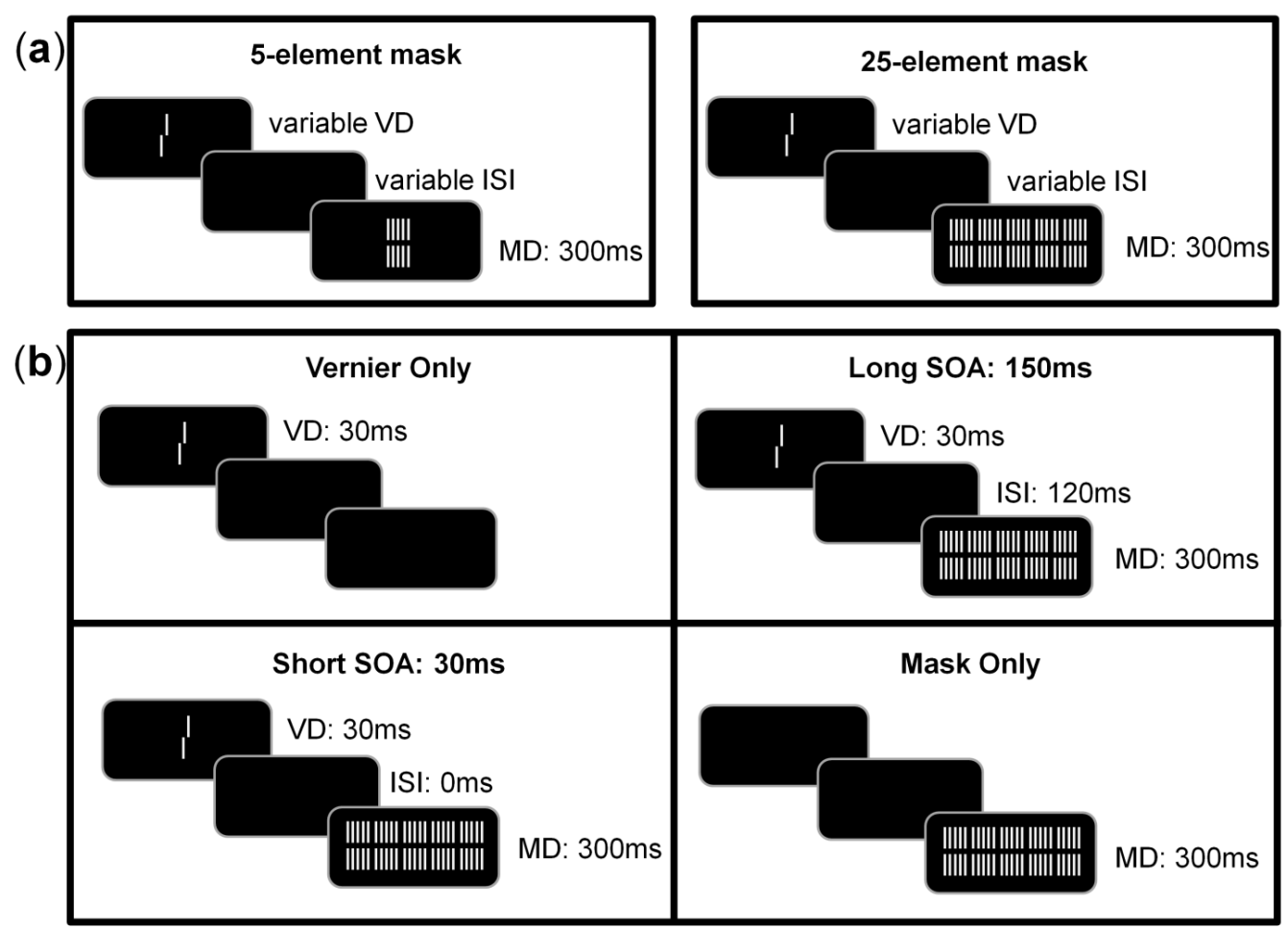

Figure S1| Stimuli display. The vernier is first presented and then followed by a grating mask. (a) left: adaptive procedure for the 5-element mask and right: 25-element mask. (b) the four condition of the EEG experiment. 
(a) $\quad \mathrm{PO7}$
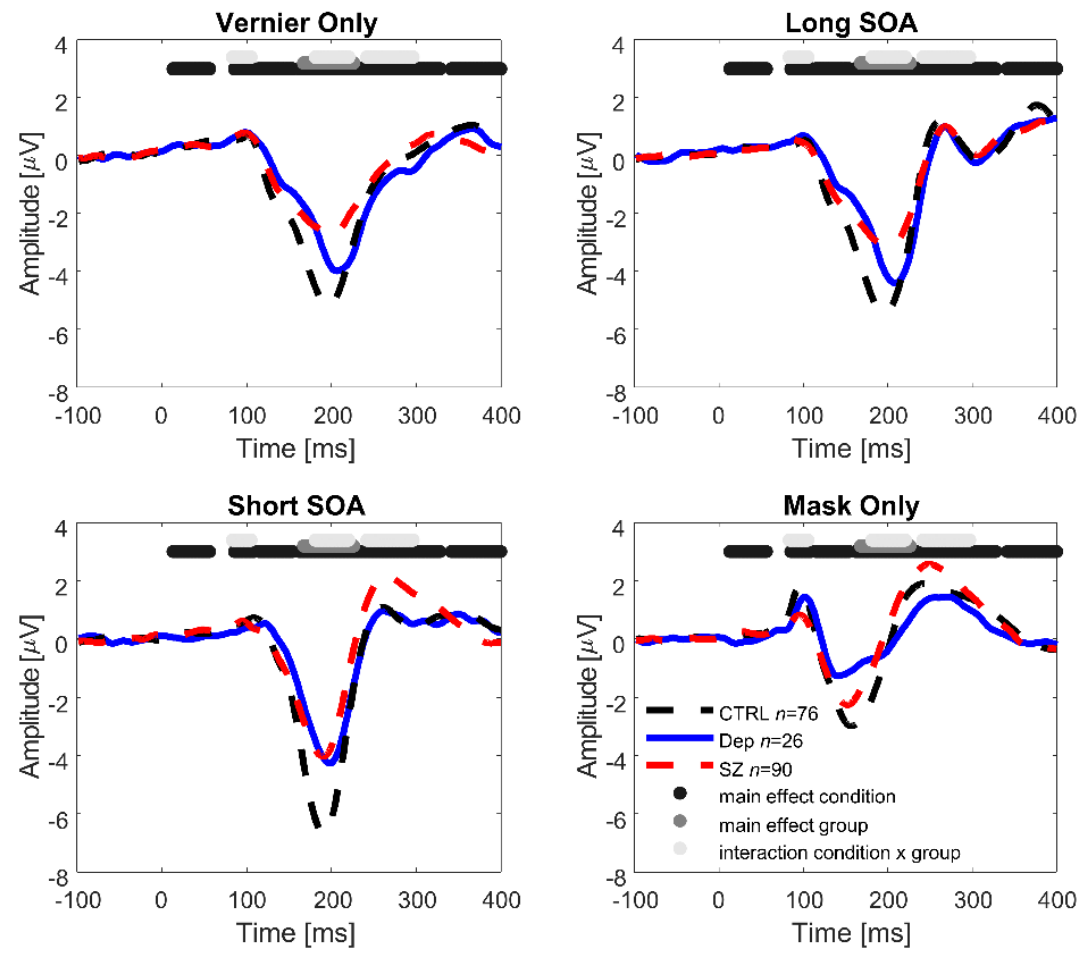

(b)

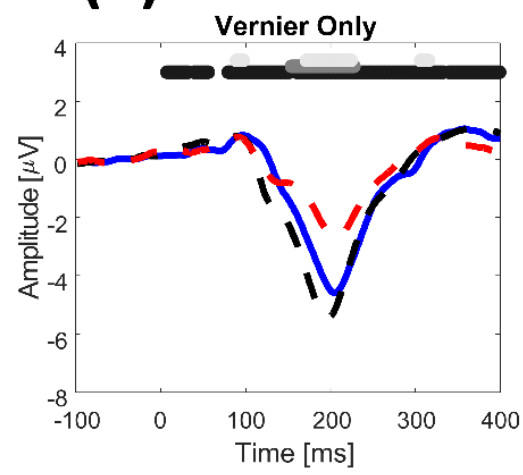

PO8
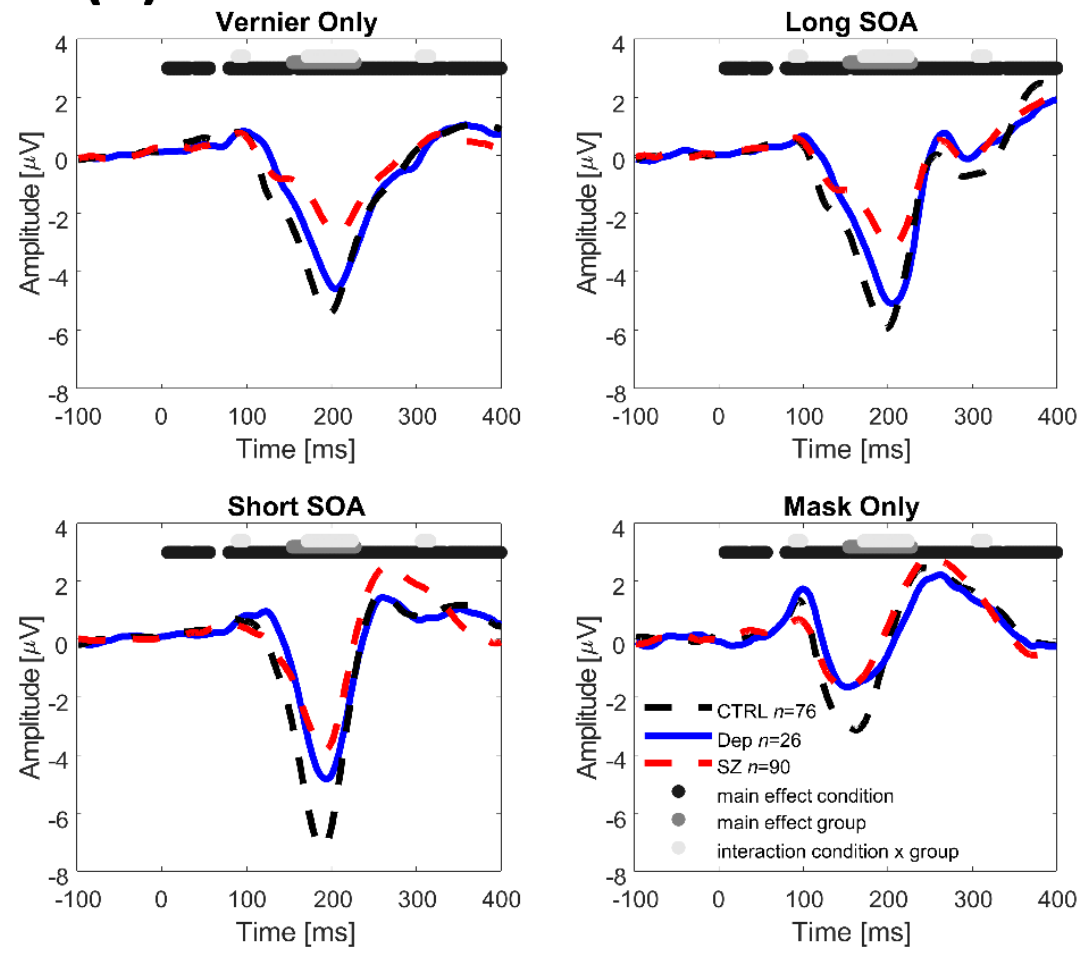

Figure S2| ERPs recorded from two occipital electrodes for the four conditions (a) PO7 and (b) PO8 
(a)
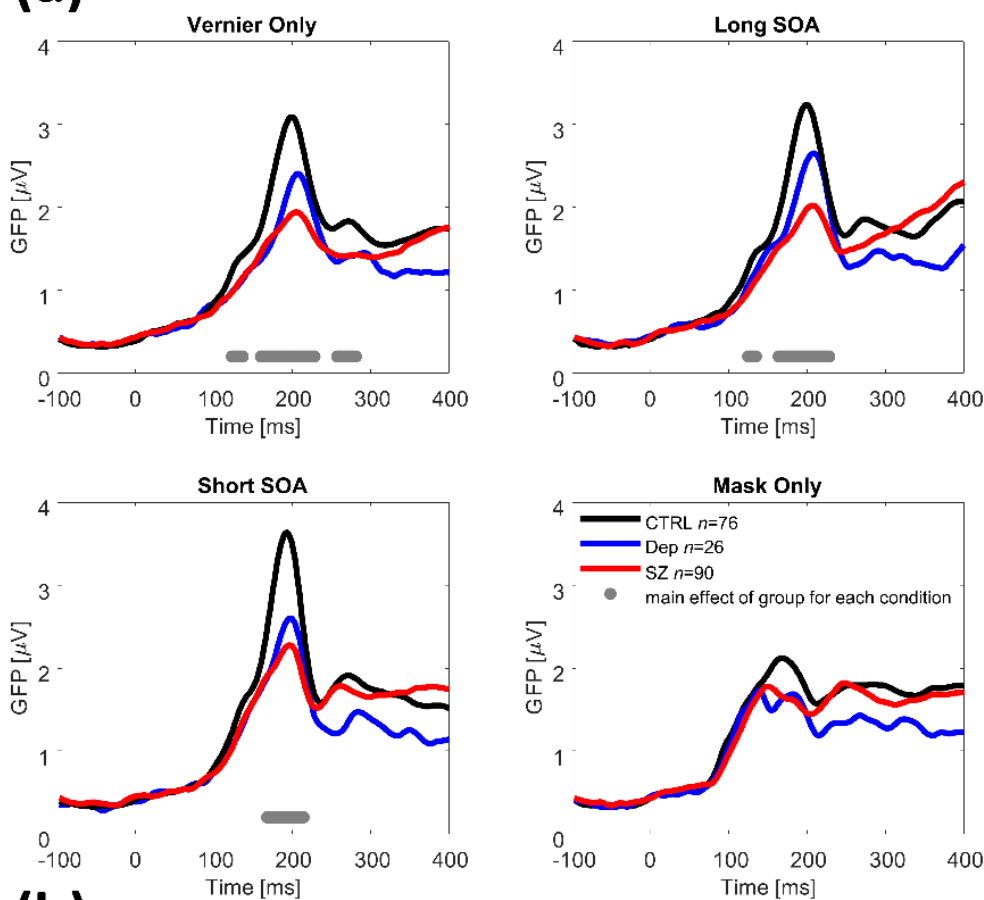

(b)
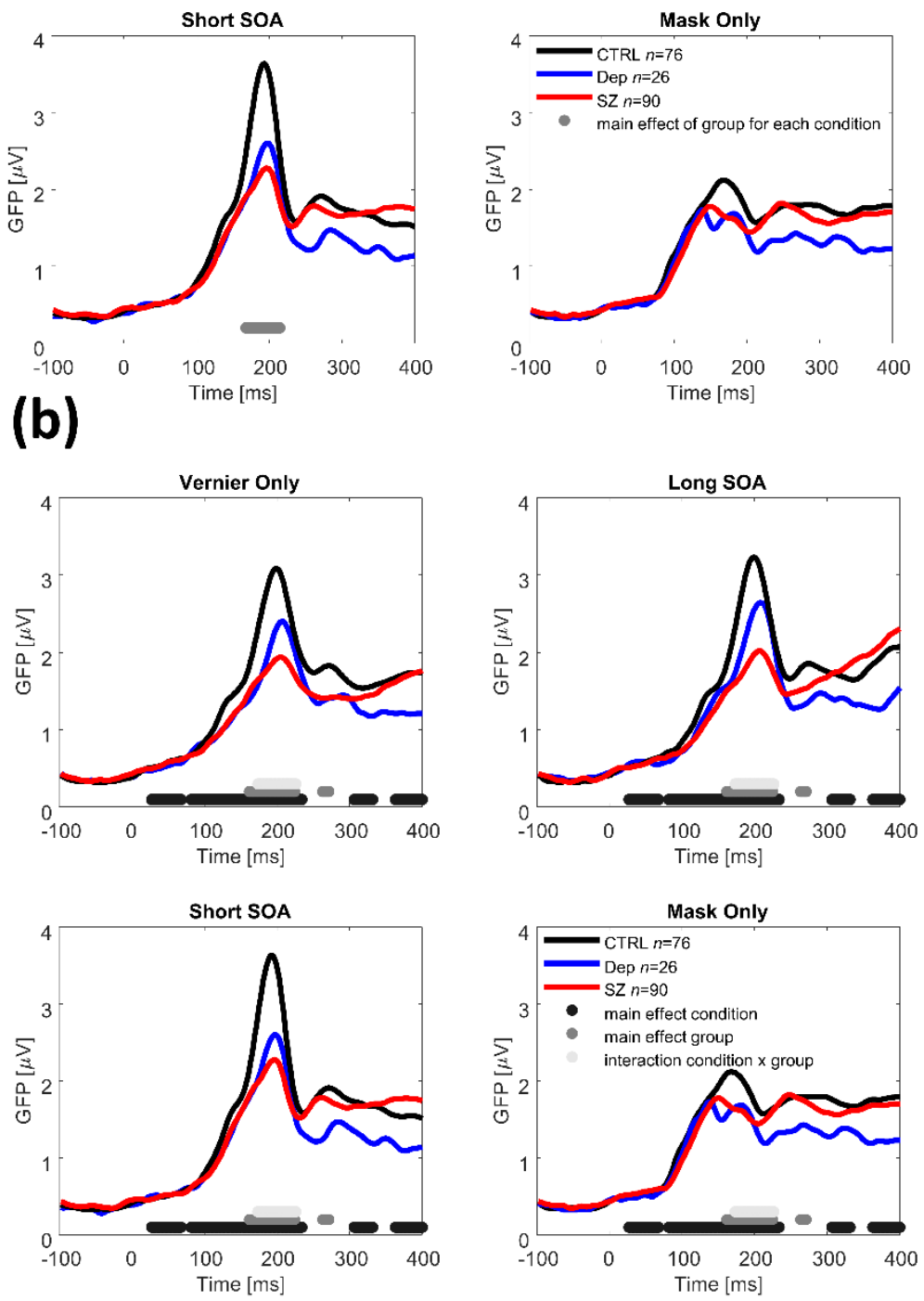

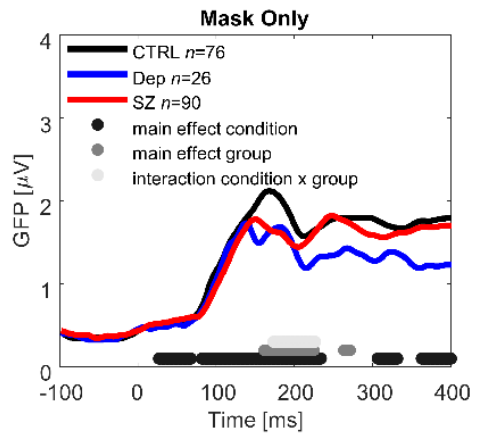

Figure S3| (a) Global Field Power for the controls (Ctrl, black) and schizophrenia patients (Sz, red) from (da Cruz et al., submitted) and depressive patients (Dep, blue). The horizontal lines indicate the significant time interval ( $p<0.05$, fdr-corrected) for the main effect of group (gray) 
for each condition independently as in (da Cruz et al., submitted). (b) Idem, but with a statistical analysis comparable to Favrod et al., 2017 and 2018. A 3 groups $x 4$ conditions rm-ANOVA is computed.

Table S1| Demographics for the depressive patients groups

\begin{tabular}{cccc}
\hline & WCST & CPT & VF \\
\hline $\boldsymbol{n}$ & 38 & 37 & 36 \\
Gender (F/M) & $25 / 13$ & $24 / 13$ & $23 / 13$ \\
Age (years) \pm SD & $34.0 \pm 10.5$ & $35.5 \pm 10.1$ & $33.4 \pm 10.2$ \\
Education (years) \pm SD & $14.5 \pm 2.6$ & $14.6 \pm 2.6$ & $14.6 \pm 2.7$ \\
Illness Duration (months) \pm & $6.7 \pm 6.4$ & $6.0 \pm 5.0$ & $6.2 \pm 5.0$ \\
SD & $31.4 \pm 6.7$ & $31.2 \pm 6.7$ & $31.4 \pm 6.7$ \\
BPRS & $15.3 \pm 5.7^{*}$ & $15.3 \pm 5.7^{\wedge}$ & $15.4 \pm 5.7^{\wedge}$ \\
Hamilton & $265.7 \pm 280.2^{* *}$ & $253.7 \pm 277.7^{* *}$ & $253.7 \pm 277.7^{\wedge \wedge}$ \\
CPZ equivalent \pm SD & $1 / 37$ & $1 / 36$ & $1 / 35$ \\
Handedness (L/R) & $1.3 \pm 0.5^{* * *}$ & $1.3 \pm 0.5^{* * *}$ & $1.3 \pm 0.5^{* * *}$ \\
Visual Acuity \pm SD & & & \\
\hline
\end{tabular}

Note: ***1 the VA from one patient was missing,

$\wedge 5, * 6$ patients were not assessed with the Hamilton scale,

$\wedge \wedge 8, * * 9$ patients were not receiving any neuroleptic medication.

Table S2| N1 amplitudes average and standard deviation of the peak maxima (around $200 \mathrm{~ms}$ ) for each population and each condition.

\begin{tabular}{|c|c|c|c|}
\hline $\begin{array}{c}\text { mean } \pm \text { SD } \\
\text { (peak latency) }\end{array}$ & Ctrl & Dep & Sz \\
\hline Vernier Only & $3.1 \pm 1.4(199 \mathrm{~ms})$ & $2.4 \pm 0.9(207 \mathrm{~ms})$ & $1.9 \pm 1.1(205 \mathrm{~ms})$ \\
\hline Long SOA & $3.2 \pm 1.4(199 \mathrm{~ms})$ & $2.6 \pm 1.0(209 \mathrm{~ms})$ & $2.0 \pm 1.0(207 \mathrm{~ms})$ \\
\hline Short SOA & $3.6 \pm 1.6(193 \mathrm{~ms})$ & $2.6 \pm 1.1(197 \mathrm{~ms})$ & $2.3 \pm 1.2(197 \mathrm{~ms})$ \\
\hline Mask Only & $2.1 \pm 1.0(168 \mathrm{~ms})$ & $1.7 \pm 0.7(138 \mathrm{~ms})$ & $1.8 \pm 0.9(150 \mathrm{~ms})$ \\
\hline
\end{tabular}


Table S3| Complementary statistical analysis: for the visual backward masking performance: adaptive masking experiment (5- and 25-element mask) and EEG experiment, for the GFP N1 peak and for the three cognitive tasks (VFT, CPT and WCST).

\begin{tabular}{|c|c|c|c|c|c|}
\hline \multicolumn{6}{|c|}{ SOA 5-element and 25-element } \\
\hline mask*group & \multicolumn{4}{|c|}{$\mathrm{F}(2,191)=3.492, \mathrm{p}=0.032, \eta^{2}=0.018$} & \multirow[t]{2}{*}{ Simple main effec } \\
\hline post-hoc & \multicolumn{2}{|c|}{ Ctrl vs Dep } & Ctrl vs Sz & Dep vs Sz & \\
\hline SOA5 & \multicolumn{2}{|c|}{$\begin{array}{c}\mathrm{t}(108)=-0.350 \\
\mathrm{~d}=-0.122 \\
\text { pholm }=0.727\end{array}$} & $\begin{array}{c}\mathrm{t}(164)=-6.701, \\
\mathrm{~d}=-0.994, \\
\mathrm{p}_{\text {holm }}<0.001\end{array}$ & $\begin{array}{c}\mathrm{t}(122)=-4.828 \\
\mathrm{~d}=-0.811 \\
\mathrm{p}_{\text {holm }}<0.001\end{array}$ & $\begin{array}{c}F(2,197)=25.90, \\
p<0.001, \eta^{2}=0.208\end{array}$ \\
\hline SOA25 & \multicolumn{2}{|c|}{$\begin{array}{c}\mathrm{t}(108)=-2.017 \\
\mathrm{~d}=-0.589 \\
\mathrm{p} \text { holm }=0.045\end{array}$} & $\begin{array}{c}\mathrm{t}(164)=-9.300, \\
\mathrm{~d}=-1.451, \\
\text { pholm }<0.001\end{array}$ & $\begin{array}{c}\mathrm{t}(122)=-5.129 \\
\mathrm{~d}=-0.858 \\
\text { pholm }<0.001\end{array}$ & $\begin{array}{c}F(2,197)=45.16 \\
p<0.001, \eta^{2}=0.314\end{array}$ \\
\hline \multicolumn{6}{|c|}{ EEG experiment } \\
\hline conditiol & & \multicolumn{4}{|c|}{$\mathrm{F}(1.788,338.005)=1285.76, \mathrm{p}<0.001, \eta^{2}=0.847$} \\
\hline VO vs LSC & & \multicolumn{4}{|c|}{$\mathrm{t}(382)=6.937, \mathrm{~d}=0.501, \mathrm{p}_{\text {holm }}<0.001$} \\
\hline VO vs SSC & & \multicolumn{4}{|c|}{$\mathrm{t}(382)=23.895, \mathrm{~d}=1.724$, pholm $<0.001$} \\
\hline VO vs M( & & \multicolumn{4}{|c|}{$\mathrm{t}(382)=80.353, \mathrm{~d}=5.799, \mathrm{p}_{\text {holm }}<0.001$} \\
\hline LSOA vs SS & & \multicolumn{4}{|c|}{$\mathrm{t}(382)=23.785, \mathrm{~d}=1.717$, pholm $<0.001$} \\
\hline LSOA vs N & & \multicolumn{4}{|c|}{$\mathrm{t}(382)=57.850, \mathrm{~d}=4.175$, pholm $<0.001$} \\
\hline SSOA vs N & & \multicolumn{4}{|c|}{$\mathrm{t}(382)=20.793, \mathrm{~d}=1.501, \mathrm{p}_{\text {holm }}<0.001$} \\
\hline \multicolumn{2}{|c|}{ condition*group } & \multicolumn{3}{|c|}{$\mathrm{F}(3.577,338.005)=21.40, \mathrm{p}<0.001, \eta^{2}=0.028$} & \multirow[t]{2}{*}{ Simple main effect } \\
\hline post-hoc & & Ctrl vs Dep & Ctrl vs Sz & Dep vs Sz & \\
\hline \multicolumn{2}{|c|}{ Vernier Only } & $\begin{array}{c}\mathrm{t}(100)=1.721, \\
\mathrm{~d}=0.454 \\
\mathrm{p}_{\text {holm }}=0.087\end{array}$ & $\begin{array}{c}\mathrm{t}(164)=5.743, \\
\mathrm{~d}=0.953, \\
\mathrm{p}_{\text {holm }}<0.001\end{array}$ & $\begin{array}{c}\mathrm{t}(114)=2.262, \\
\mathrm{~d}=0.426, \\
\mathrm{p}_{\text {holm }}=0.050\end{array}$ & $\begin{array}{c}F(2,189)=16.59 \\
p<0.001, \eta^{2}=0.149\end{array}$ \\
\hline \multicolumn{2}{|c|}{ Long SOA } & $\begin{array}{c}\mathrm{t}(100)=1.304 \\
\mathrm{~d}=0.394 \\
\text { pholm }=0.194\end{array}$ & $\begin{array}{c}\mathrm{t}(164)=6.931, \\
\mathrm{~d}=1.094, \\
\mathrm{p}_{\mathrm{holm}}<0.001\end{array}$ & $\begin{array}{c}\mathrm{t}(114)=3.519, \\
\mathrm{~d}=0.658 \\
\text { pholm }=0.001\end{array}$ & $\begin{array}{c}F(2,189)=24.96 \\
p<0.001, \eta^{2}=0.209\end{array}$ \\
\hline \multicolumn{2}{|c|}{ Short SOA } & $\begin{array}{c}\mathrm{t}(100)=0.993 \\
\mathrm{~d}=0.218, \\
\mathrm{p}_{\mathrm{holm}}=0.322\end{array}$ & $\begin{array}{c}\mathrm{t}(164)=7.712, \\
\mathrm{~d}=1.235 \\
\mathrm{p}_{\text {holm }}<0.001\end{array}$ & $\begin{array}{c}\mathrm{t}(114)=4.383 \\
\mathrm{~d}=0.970 \\
\mathrm{p}_{\text {holm }}<0.001\end{array}$ & $\begin{array}{c}F(2,189)=31.78, \\
p<0.001, \eta^{2}=0.252\end{array}$ \\
\hline \multicolumn{2}{|c|}{ Mask Only } & \multicolumn{4}{|c|}{$F(2,189)=0.082, p=0.921, \eta^{2}=0$} \\
\hline \multicolumn{6}{|c|}{$200 \mathrm{~ms}$ N1 peak } \\
\hline \multicolumn{2}{|c|}{ condition } & \multicolumn{4}{|c|}{$\mathrm{F}(2.108,398.337)=60.01, \mathrm{p}<0.001, \eta^{2}=0.221$} \\
\hline \multicolumn{2}{|c|}{ VO vs LSOA } & \multicolumn{4}{|c|}{$\mathrm{t}(382)=-4.349, \mathrm{~d}=-0.314$, pholm $<0.001$} \\
\hline \multicolumn{2}{|c|}{ VO vs SSOA } & \multicolumn{4}{|c|}{$\mathrm{t}(382)=-7.160, \mathrm{~d}=-0.517$, pholm $<0.001$} \\
\hline \multicolumn{2}{|c|}{ VO vs MO } & \multicolumn{4}{|c|}{$\mathrm{t}(382)=6.837, \mathrm{~d}=0.493, \mathrm{p}_{\text {holm }}<0.001$} \\
\hline \multicolumn{2}{|c|}{ LSOA vs SSOA } & & $t(382)=-4.4$ & $3, \mathrm{~d}=-0.318, \mathrm{p}_{\text {holn }}$ & 001 \\
\hline LSOA vs N & & & $t(382)=8.2$ & $5, \mathrm{~d}=0.596, \mathrm{p}_{\text {holm }}$ & \\
\hline SSOA vs N & & & $\mathrm{t}(382)=11$. & $78, \mathrm{~d}=0.807, \mathrm{p}_{\text {holn }}$ & 001 \\
\hline & & & VFT & & \\
\hline category & & & $\mathrm{F}(1,104)=6$ & $.644, \mathrm{p}<0.001, \eta^{2}$ & 374 \\
\hline
\end{tabular}




\begin{tabular}{|c|c|c|c|c|}
\hline cat I vs cat II & \multicolumn{4}{|c|}{$\mathrm{t}(212)=-9.149, \mathrm{~d}=-0.884$, pholm $<0.001$} \\
\hline category*group & \multicolumn{3}{|c|}{$\mathrm{F}(2,104)=3.753, \mathrm{p}=0.027, \eta^{2}=0.042$} & \multirow[t]{2}{*}{ Simple main effect } \\
\hline post-hoc & Ctrl vs Dep & Ctrl vs Sz & Dep vs Sz & \\
\hline cat $I$ & $\begin{array}{c}\mathrm{t}(82)=2.208, \\
\mathrm{~d}=0.476, \\
\mathrm{p}_{\mathrm{holm}}=0.087\end{array}$ & $\begin{array}{c}\mathrm{t}(69)=2.213 \\
\mathrm{~d}=0.550 \\
\mathrm{p}_{\mathrm{holm}}=0.087\end{array}$ & $\begin{array}{c}\mathrm{t}(57)=0.279 \\
\mathrm{~d}=0.079 \\
\mathrm{p}_{\text {holm }}=0.781\end{array}$ & $\begin{array}{c}\mathrm{F}(2,104)=3.561, \mathrm{p}=0.0 \\
2, \eta^{2}=0.064\end{array}$ \\
\hline cat II & $\begin{array}{c}\mathrm{t}(82)=3.172, \\
\mathrm{~d}=0.745, \\
\mathrm{p}_{\text {holm }}=0.004\end{array}$ & $\begin{array}{c}\mathrm{t}(69)=4.790 \\
\mathrm{~d}=1.253, \\
\mathrm{p}_{\mathrm{holm}}<0.001\end{array}$ & $\begin{array}{c}\mathrm{t}(57)=1.931 \\
\mathrm{~d}=0.462, \\
\mathrm{p}_{\text {holm }}=0.056\end{array}$ & $\begin{array}{l}F(2,104)=12.589, \\
p<0.001, \eta^{2}=0.195\end{array}$ \\
\hline \multicolumn{5}{|c|}{ WCST } \\
\hline measure & \multicolumn{4}{|c|}{$\mathrm{F}(1.304,258.141)=1156.75, \mathrm{p}<0.001, \eta^{2}=0.841$} \\
\hline cat vs corr & \multicolumn{4}{|c|}{$\mathrm{t}(400)=-73.340, \mathrm{~d}=-5.173, \mathrm{p}_{\text {holm }}<0.001$} \\
\hline cat vs err & \multicolumn{4}{|c|}{$\mathrm{t}(400)=-17.286, \mathrm{~d}=-1.219, \mathrm{p}_{\text {holm }}<0.001$} \\
\hline cat vs pers & \multicolumn{4}{|c|}{$\mathrm{t}(400)=-2.895, \mathrm{~d}=-0.204, \mathrm{p}_{\text {holm }}=0.004$} \\
\hline corr vs err & \multicolumn{4}{|c|}{$\mathrm{t}(400)=24.902, \mathrm{~d}=1.756, \mathrm{p}_{\mathrm{holm}}<0.001$} \\
\hline corr vs pers & \multicolumn{4}{|c|}{$\mathrm{t}(400)=37.545, \mathrm{~d}=2.648$, pholm $<0.001$} \\
\hline err vs pers & \multicolumn{4}{|c|}{$\mathrm{t}(400)=21.769, \mathrm{~d}=1.535$, pholm $<0.001$} \\
\hline
\end{tabular}

Table S4| Statistical analysis of the demographical data: Gender is unmatched comparing schizophrenia patients with controls and depressive patients. Similarly, education differs in schizophrenia patients compared to controls and depressive patients. Finally, visual acuity is higher for controls compared to both group of patients.

\begin{tabular}{|c|c|c|c|c|c|c|c|}
\hline & \multirow[t]{2}{*}{ Controls } & \multirow{2}{*}{$\begin{array}{c}\text { Depression } \\
\text { (adaptive) }\end{array}$} & \multirow[t]{2}{*}{ Schizophrenia } & \multirow[t]{2}{*}{ Statistics } & \multicolumn{3}{|c|}{ Post-hoc ( $p$-values) } \\
\hline & & & & & $\begin{array}{c}\text { Controls } \\
\text { vs } \\
\text { Depression }\end{array}$ & $\begin{array}{c}\text { Controls vs } \\
\text { Schizophrenia }\end{array}$ & $\begin{array}{l}\text { Depressive vs } \\
\text { Schizophrenia }\end{array}$ \\
\hline Gender $(\mathrm{F} / \mathrm{M})$ & $34 / 42$ & $22 / 12$ & $15 / 75$ & $\begin{array}{c}\chi^{2}(2)=29.439, \\
P<0.001\end{array}$ & 0.053 & $<0.001$ & $<0.001$ \\
\hline $\begin{array}{l}\text { Age (years) } \pm \\
\text { SD }\end{array}$ & $34.9 \pm 8.1$ & $32.6 \pm 10.0$ & $35.8 \pm 8.8$ & $\begin{array}{c}F(2,197)=1.300, \\
P=0.275\end{array}$ & & & \\
\hline $\begin{array}{l}\text { Education } \\
\text { (years) } \pm \text { SD }\end{array}$ & $15.0 \pm 2.8$ & $14.8 \pm 2.6$ & $13.2 .0 \pm 2.7$ & $\begin{array}{c}F(2,197)=9.358, \\
P<0.001\end{array}$ & 0.677 & $<0.001$ & 0.013 \\
\hline $\begin{array}{l}\text { Handedness } \\
(\mathrm{R} / \mathrm{L})\end{array}$ & $71 / 5$ & $33 / 1$ & $86 / 4$ & $\begin{array}{c}\chi^{2}(2)=0.599 \\
P=0.741\end{array}$ & & & \\
\hline $\begin{array}{l}\text { Visual acuity } \\
\pm \text { SD }\end{array}$ & $1.6 \pm 0.4$ & $1.4 \pm 0.4$ & $1.5 \pm 0.4$ & $\begin{array}{c}F(2,197)=6.046, \\
P=0.003\end{array}$ & 0.011 & 0.011 & 0.495 \\
\hline
\end{tabular}

Table S5 $\mid$ Two-way repeated measures ANOVA with factors mask (5 and 25 elements grating) and group (controls, depression, ands) with visual acuity (VA), education (Ed), and gender as co-variates

\begin{tabular}{|c|c|c|c|c|c|}
\hline Effect & $F$-value & df1 & df2 & $p$-value & $\eta^{2}$ \\
\hline Mask & $\mathbf{1 3 . 3 2 0}$ & $\mathbf{1}$ & $\mathbf{1 9 4}$ & $<\mathbf{0 . 0 0 1}$ & $\mathbf{0 . 0 6 1}$ \\
\hline Mask x Group & 3.584 & 2 & 194 & 0.030 & 0.033 \\
\hline Mask x VA & 1.493 & 1 & 194 & 0.223 & 0.007 \\
\hline Mask x Ed & 0.597 & 1 & 194 & 0.441 & 0.003 \\
\hline
\end{tabular}




\begin{tabular}{|c|c|c|c|c|c|}
\hline Mask x Gender & 1.390 & 1 & 194 & 0.240 & 0.006 \\
\hline Group & $\mathbf{3 5 . 7 9 0}$ & $\mathbf{2}$ & $\mathbf{1 9 4}$ & $<\mathbf{0 . 0 0 1}$ & $\mathbf{0 . 2 5 9}$ \\
\hline VA & 1.164 & 1 & 194 & 0.282 & 0.004 \\
\hline Ed & 1.012 & 1 & 194 & 0.316 & 0.004 \\
\hline Gender & $\mathbf{8 . 0 8 6}$ & $\mathbf{1}$ & $\mathbf{1 9 4}$ & $\mathbf{0 . 0 0 5}$ & $\mathbf{0 . 0 2 9}$ \\
\hline
\end{tabular}

Table S6| Simple Main effect of group, for each of the two masking conditions (5 and 25 elements grating), with gender, education ( $E d)$, and visual acuity (VA) as co-variates, Bonferroni-Holm corrected for multiple comparisons.

\begin{tabular}{|c|c|c|c|c|c|c|}
\hline Mask & Effect & $F$-value & df1 & df2 & $p$-value & $\eta^{2}$ \\
\hline \multirow{3}{*}{5 grating } & Group & $\mathbf{2 3 . 9 7 0}$ & $\mathbf{2}$ & $\mathbf{1 9 4}$ & $<\mathbf{0 . 0 0 1}$ & $\mathbf{0 . 1 9 1}$ \\
\cline { 2 - 8 } & Gender & $\mathbf{6 . 2 1 3}$ & $\mathbf{1}$ & $\mathbf{1 9 4}$ & $\mathbf{0 . 0 1 4}$ & $\mathbf{0 . 0 2 5}$ \\
\cline { 2 - 8 } & Ed & 1.041 & 1 & 194 & 0.309 & 0.004 \\
\cline { 2 - 8 } & VA & 1.556 & 1 & 194 & 0.214 & 0.006 \\
\hline \multirow{3}{*}{25 grating } & Group & 42.006 & $\mathbf{2}$ & $\mathbf{1 9 4}$ & $<\mathbf{0 . 0 0 1}$ & 0.293 \\
\cline { 2 - 8 } & Gender & 7.586 & $\mathbf{1}$ & $\mathbf{1 9 4}$ & $\mathbf{0 . 0 0 6}$ & 0.026 \\
\cline { 2 - 8 } & Ed & 0.535 & 1 & 194 & 0.465 & 0.002 \\
\cline { 2 - 8 } & VA & 0.266 & 1 & 194 & 0.606 & $<0.001$ \\
\hline
\end{tabular}

Table S7| Post-hoc pairwise group comparisons of the mean SOAs, Bonferroni-Holm corrected, for each 2 masking conditions (5 and 25 elements grating), with gender, education (Ed), and visual acuity (VA) as co-variates.

\begin{tabular}{|c|c|c|c|c|c|c|c|}
\hline Mask & Group I & Group II & $\begin{array}{c}\text { Mean } \\
\text { Difference }\end{array}$ & SE & $t$-value & $p$-value & d \\
\hline \multirow{3}{*}{5 grating } & Controls & Depressive & 5.664 & 17.058 & 0.332 & 0.740 & 0.118 \\
\hline & & Schizophrenia & -87.121 & 13.908 & -6.264 & $<0.001$ & 1.019 \\
\hline & Depressive & Schizophrenia & -92.785 & 17.409 & -5.330 & $<0.001$ & 0.950 \\
\hline \multirow{3}{*}{25 grating } & Controls & Depressive & -13.900 & 9.742 & -1.427 & 0.155 & 0.424 \\
\hline & & Schizophrenia & -70.792 & 7.943 & -8.912 & $<0.001$ & 1.527 \\
\hline & Depressive & Schizophrenia & -56.892 & 9.943 & -5.722 & $<0.001$ & 1.018 \\
\hline
\end{tabular}

